\title{
STRESS ANALYSIS OF POLARIZATION MAINTAINING OPTICAL FIBERS BY THE FINITE ELEMENT METHOD
}

\author{
M. H. Aly \\ Faculty of Engineering, University of Alexandria, Alexandria 21544, Egypt,
}

A. S. Farahat, M. S. Helmi and M. Farhoud

Faculty of Science, University of Alexandria, Alexandria, Egypt

\begin{abstract}
Stress-induced birefringence in single mode polarization maintaining optical fibers has been investigated using the finite element method. The modal birefringence caused by external forces in the Panda and the Side Tunnel fibers are calculated. It is found that the modal birefringence is directly proportional to the radial distance from the fiber center. As expected, the modal birefringence vanishes with the variation in the magnitude of the applied external loads.
\end{abstract}

Key Words: Birefringence, Polarization, Panda Fiber, Side-Pit Fiber, Finite Element Method.

\section{INTRODUCTION}

Polarization maintaining (PM) optical fibers are of great importance in optical coherent communications and some optical fiber sensor applications ${ }^{[1]}$. The fibers that preserve the state of polarization of the guided light can be achieved by destroying the circular symmetry of the refractive index distribution in the core region. However, the birefringence properties of this kind of fiber are much more limited when compared with what can be achieved with stress induced birefringence. Recently, a variety of structures has been proposed for the polarization maintaining fibers with stress induced birefringence, most of which are designed to maintain a differential stress in the fiber core region ${ }^{[1]}$. There are many such successful fiber structure designs, such as Panda, Bow Tie, Side Tunnel and Side Pit fibers ${ }^{[1]}$. Shih $^{[2]}$ described a new fiber manufacturing technique called preform deformation. Using this technique, a new kind of PM fiber with a circular core, an elliptical stress applying cladding, and an elliptical jacket can be made. This has been studied experimentally by D. Marcuse et al. ${ }^{[3]}$. In our work, full advantage of the finite element method is taken to develop a numerical study of the birefringence due to the effects of external stresses in both Side Pit and the Side Tunnel fibers. The birefringence PM fibers manufactured through preform deformation are analyzed. From the calculated stress distribution in the fiber, the refractive index tensor change is obtained.

The finite element method is an approximate treatment of physical problems, defined, for example, by differential equations with boundary conditions, or by variation principles. Compared to other approximate methods it is successful when the domain of the problem has a complicated shape or when the function involved behaves differently in different parts of the domain. The domain is represented approximately by a collection of finite numbers of connected subdomains of simple shape (e.g., triangles), called finite elements.

The finite element method will to be applied in this paper for the determination of the stress distribution and the modal birefringence, due to the effects of external forces acting on the fiber cross section, for the Panda and the Side Tunnel type fibers. The fiber cross section is first divided into "elements". In this research, a triangular element is used. A linear system of equations can be established according to the principles of the finite element method, and then the stress distribution and, further, the refractive index tensor distribution may be calculated in each element. The details of the use of the finite element method can be found in the literature $^{[4-5]}$.

\section{FORMULATION OF THE PROBLEM BY THE FINITE ELEMENT METHOD}

\subsection{Matrix Solution Techniques}

Solving the waveguide problem by the finite element method, the key factor affecting storage requirements and computational efforts is the choice of the algorithm to solve the matrix equation. The advantage of higherorder basis functions for the fields is that they give a more accurate solution. But it involves an increased programming effort, particularly when considering anisotropic materials, in the finite element, and penalty functions ${ }^{[6]}$. Another advantage of the higher order polynomials (for given matrix order) is that it increases the density of the matrix. It should be noted the tradeoff optimum choice between low and high order polynomials depends on the matrix algorithm used.

\subsection{Principle of Minimum Potential Energy}

The basic principle of the finite element method is that a continuum (the total structure) can be modeled analytically by its subdivision into regions.

The principle of minimum potential energy can be used following Ref. [1]. Among all displacements of admissible form, those that satisfy the equilibrium 
conditions make the potential energy assume a stationary (minimum) value. The total potential energy of a structure can be expressed as a strain energy, U, plus the potential energy, V, of the applied loads, i.e.,

$\Pi=\mathrm{U}+\mathrm{V}$

The strain energy $U$ of a complete structure consisting of $\mathrm{N}$ elements is simply the sum of the $\mathrm{N}$ element strain energies

$$
\left.U=\sum_{\mathrm{e}=1}^{\mathrm{N}} \mathrm{U}^{\mathrm{e}}=\sum_{\mathrm{e}=1}^{\mathrm{N}}\left(\frac{1}{2}\left\{\mathrm{u}^{\mathrm{e}}\right\}\left[\mathrm{K}^{\mathrm{e}}\right]\left\{\mathrm{u}^{\mathrm{e}}\right\}-\left\{\mathrm{u}^{\mathrm{e}}\right\} \mathrm{f}_{\mathrm{T}}^{\mathrm{f}} \mathrm{e}\right\}\right)
$$

where the superscript $t$ is the operator to traznspose the vector or the matrix, and $\left\{\mathrm{u}^{\mathrm{e}}\right\},\left[\mathrm{K}^{\mathrm{e}}\right]$, and $\left\{\mathrm{f}_{\mathrm{T}}^{\mathrm{e}}\right\}$ are the vector of nodal points displacements, the stiffness matrix, and the initial force vector in the eth element, respectively. Equation (2) is rearranged to construct the global equation as:

$\mathrm{U}=\frac{1}{2}\{\mathrm{u}\}^{\mathrm{t}}[\mathrm{K}]\{\mathrm{u}\}-\{\mathrm{u}\}^{\mathrm{t}}\left\{\mathrm{f}_{\mathrm{T}}\right\}$,

where $\{\mathrm{u}\},[\mathrm{K}]$, and $\left\{\mathrm{f}_{\mathrm{T}}\right\}$ denote the global nodal point displacement vector, the global stiffness matrix and the global initial force vector, respectively.

The potential energy, $\mathrm{V}$, due to external load is expressed by:

$\mathrm{V}=-\{\mathrm{u}\}^{\mathrm{t}}\left\{\mathrm{f}_{\mathrm{L}}\right\}$

where $\left\{f_{L}\right\}$ is the global load vector acting on each nodal point. Inserting expressions for $\mathrm{U}$ and $\mathrm{V}$ from $\mathrm{Eq}$. (3) and Eq. (4) into Eq. (1), one can get:

$\Pi=\frac{1}{2}\{u\}^{t}[\mathrm{~K}]\{\mathrm{u}\}-\{\mathrm{u}\}^{\mathrm{t}}\left\{\mathrm{f}_{\mathrm{T}}\right\}-\{\mathrm{u}\}^{\mathrm{t}}\left\{\mathrm{f}_{\mathrm{L}}\right\}$,

Applying to this the necessary condition for minimum energy, i.e.,

$\frac{\partial \Pi}{\partial\{\mathrm{u}\}}=[\mathrm{K}]\{\mathrm{u}\}-\left\{\mathrm{f}_{\mathrm{T}}\right\}-\left\{\mathrm{f}_{\mathrm{L}}\right\}=0$,

$[\mathrm{K}]\{\mathrm{u}\}=\left\{\mathrm{f}_{\mathrm{T}}\right\}+\left\{\mathrm{f}_{\mathrm{L}}\right\}$

This equation gives the displacement at all nodal points of the fiber under external forces. Once the global nodal point displacement vector, $\{\mathrm{u}\}$, is determined, stress in each element is given by ${ }^{[1]}$ :

$$
\left\{\sigma^{\mathrm{e}}\right\}=\left[\mathrm{D}^{\mathrm{e}}\right]\left(\left[\mathrm{B}^{\mathrm{e}}\right]\left\{\mathrm{u}^{\mathrm{e}}\right\}-\left\{\epsilon_{\mathrm{o}}^{\mathrm{e}}\right\}\right) \text {. }
$$

with $\mathrm{e}=1,2,3 \ldots \mathrm{N}$, where $\left[\mathrm{D}^{\mathrm{e}}\right],\left[\mathrm{B}^{\mathrm{e}}\right]$, and $\left\{\in_{\mathrm{o}}\right\}$ are the material stiffness matrix, the matrix relating nodal displacements to strain field, and the initial strain vector in the eth element, respectively.

\section{DERIVATION OF STRAIN ENERGY}

As in the case of optical fibers, when the dimension of the structure in one direction (the z-direction) is very large in comparison with the other two transverse directions ( $\mathrm{x}-$ and $\mathrm{y}-)$ and the applied forces act in the $\mathrm{x}-$ $y$ plane and do not vary in the z-direction, the problem becomes a "plane strain problem" $"$.

In the plane strain problem, the longitudinal strain $\epsilon_{\mathrm{z}}$ is zero, as are the shear strains $\square_{\mathrm{yz}}$ and $\square_{\mathrm{zx}}$. Introducing the condition on $\epsilon_{\mathrm{z}}$ into the relevant strain-stress equation we get:

$$
\sigma_{x}=\frac{E}{(1+v)(1-2 v)}\left\{(1-v) \in_{x}+v \in_{y}\right\}-\frac{\alpha E \Delta T}{(1-2 v)}
$$

$\sigma_{y}=\frac{E}{(1+v)(1-2 v)}\left\{v \in_{x}+(1-v) \in_{y}\right\}-\frac{\alpha E \Delta T}{(1-2 v)}$

$\tau_{x y}=\frac{E}{2(1+v)} \gamma_{x y}$,

where $\sigma_{\mathrm{x}}, \sigma_{\mathrm{y}}$ and $\epsilon_{\mathrm{x}}, \in_{\mathrm{y}}$ denote the normal stresses and strains, respectively, and $\tau_{x y}$, and $\gamma_{x y}$ are the shear stress and strain, respectively, $\mathrm{E}$ is the elastic modulus, $v$ is Poisson's ratio, $\alpha$ is the thermal expansion coefficient, and $\Delta \mathrm{T}$ is the temperature change (negative on cooling). These equations are expressed in the form:

$\{\sigma\}=[D]\left(\{\in\}-\left\{\in_{o}\right\}\right)$,

where $\{\sigma\}$ and $\{\varepsilon\}$ denote the stress and strain vectors, respectively, defined as:

$\{\sigma\}=\left(\begin{array}{c}\sigma_{x} \\ \sigma_{y} \\ \tau_{x y}\end{array}\right)$,

and

$\{\in\}=\left(\begin{array}{c}\epsilon_{\mathrm{x}} \\ \epsilon_{\mathrm{y}} \\ \gamma_{\mathrm{xy}}\end{array}\right)$,

$\left\{\in_{0}\right\}$ denotes the initial strain, where:

$\epsilon_{\mathrm{o}}=(1+v) \alpha \Delta \mathrm{T}\left(\begin{array}{l}1 \\ 1 \\ 0\end{array}\right)$,

and $[\mathrm{D}]$ is the material stiffness matrix given by ${ }^{[1]}$ :

$[D]=\frac{E}{(1+v)(1-2 v)}\left(\begin{array}{ccc}(1-v), & v, & 0, \\ v, & (1-v), & 0, \\ 0, & 0, & (1-2 v) / 2\end{array}\right)$.

The strain energy in plain strain is given by ${ }^{[1]}$ :

$\mathrm{U}=\frac{\mathrm{L}}{2} \iint_{\mathrm{S}}\{\sigma\}^{\mathrm{t}}\left(\{\in\}-\left\{\epsilon_{\mathrm{o}}\right\}\right) \mathrm{dxdy}$,

where $\mathrm{L}$ is the length of the optical fiber and the integral is carried out over any region $\mathrm{S}$ under consideration. In 
this paper a two-dimensional triangular element is used to determine the variation of stresses in optical fibers. The formulation of this element is well developed and described in many references ${ }^{[6]}$. The $\mathrm{e}^{\text {th }}$ element stiffness matrix and initial force vector are:

$$
\begin{aligned}
& {\left[K^{e}\right]=\operatorname{LS}\left[\mathrm{B}^{\mathrm{e}}\right]^{\mathrm{t}}\left[\mathrm{D}^{\mathrm{e}}\right]\left[\mathrm{B}^{\mathrm{e}}\right],} \\
& \left\{f_{T}^{e}\right\}=\operatorname{LS}\left[\mathrm{B}^{\mathrm{e}}\right]^{\mathrm{t}}\left[\mathrm{D}^{\mathrm{e}}\right]\left\{\in \in_{\mathrm{o}}^{\mathrm{e}}\right\},
\end{aligned}
$$

$\mathrm{L}$ is the fiber length and $\left[\mathrm{k}^{\mathrm{e}}\right]$ and $\left\{\mathrm{f}_{\mathrm{T}}^{\mathrm{e}}\right\}$ denote the element stiffness matrix and the element initial force vector, respectively.

\section{ACCURACY OF THE FINITE ELEMENT ANALYSIS}

Before conducting the stress analysis of noncircular core fibers, the accuracy of the finite element analysis itself is first investigated.

We can estimate the amount of error by comparing the finite element solution with the analytical solutions that show the same behavior.

1. Thermal stress: This is the problem of thermal stress developing in long concentric cylinders of different materials, which are joined together at some initial temperature and their temperature is then changed. This has been treated by B.M. Azizur et $\mathrm{al}^{[7]}$.

Stress distributions in the cylinder are given as:

$$
\begin{aligned}
& \sigma_{\mathrm{r}}=\left\{\frac{\left(\mathrm{b}^{2}-\mathrm{a}^{2}\right)}{2 \mathrm{~b}^{2}} \frac{\left(\alpha_{2}-\alpha_{1}\right)}{(1-v)} \mathrm{E} \Delta \mathrm{T}, \quad(0 \leq \mathrm{r} \leq \mathrm{a})\right. \\
& =\left\{\frac{a^{2}}{2 b^{2}} \frac{\left(\alpha_{2}-\alpha_{1}\right)}{(1-v)} E \Delta T\left(\frac{b^{2}}{r^{2}}-1\right),(0 \leq r \leq b)\right. \\
& \sigma_{\theta}=\left\{\frac{\left(b^{2}-a^{2}\right)}{2 b^{2}} \frac{\left(\alpha_{2}-\alpha_{1}\right)}{(1-v)} E \Delta T,(0 \leq r \leq a)\right. \\
& =\left\{-\frac{a^{2}}{2 b^{2}} \frac{\left(\alpha_{2}-\alpha_{1}\right)}{(1-v)} E \Delta T\left(\frac{b^{2}}{r^{2}}+1\right),(0 \leq r \leq b)\right.
\end{aligned}
$$

where $\mathrm{a}$ and $\mathrm{b}$ are respectively the inner and outer radii of the concentric cylinder, $\mathrm{E}, \mathrm{v}$, and $\Delta \mathrm{T}$ are elastic modules, Poisson's ratio and temperature change in the medium, and $\alpha_{1}$ and $\alpha_{2}$ are the thermal expansion coefficients of the mediums 1 and 2, respectively.

2- Stress by loads: Stress distributions on the x-axis induced by the force $\mathrm{W}_{\mathrm{o}}$ acting on the cylinder along the vertical $y$ - axis direction of the cylinder are expressed as:
$\sigma_{x}=\frac{W_{o}}{\Pi b}\left[1-\frac{4 b^{2} x^{2}}{\left(b^{2}+x^{2}\right)^{2}}\right]$,

$\sigma_{y}=-\frac{W_{o}}{\Pi b}\left[\frac{4 b^{4}}{\left(b^{2}+x^{2}\right)^{2}}-1\right]$,

3- Stress fields by finite element analysis:

One-quarter of the entire cross section is covered by elements, since the optical fiber under consideration has two symmetry lines, one along the $\mathrm{x}$-axis and the other along the y-axis. Typically, we used 140 elements and 93 nodal points in this section. The error in the results obtained by the finite element analysis is less than 4 percent of the analytical results ${ }^{[1]}$.

\section{RESULTS AND DISCUSSION}

A computer program coded in MATLAB is used for the analysis of the birefringence properties of optical fibers using the finite element method.

The structure of the Side Tunnel and Panda fibers is shown in Figs. 1 and 2. The fiber parameters used in this study are shown in Table 1.

Taking into consideration that, in the Side Tunnel fiber, $d$ is the distance from the nearest edge of SAZ to the core center and all the refractive indices $\mathrm{n}_{1}, \mathrm{n}_{2}$, and $\mathrm{n}_{3}$ have no effect on the strain.

Fig. 1 Side Tunnel fiber

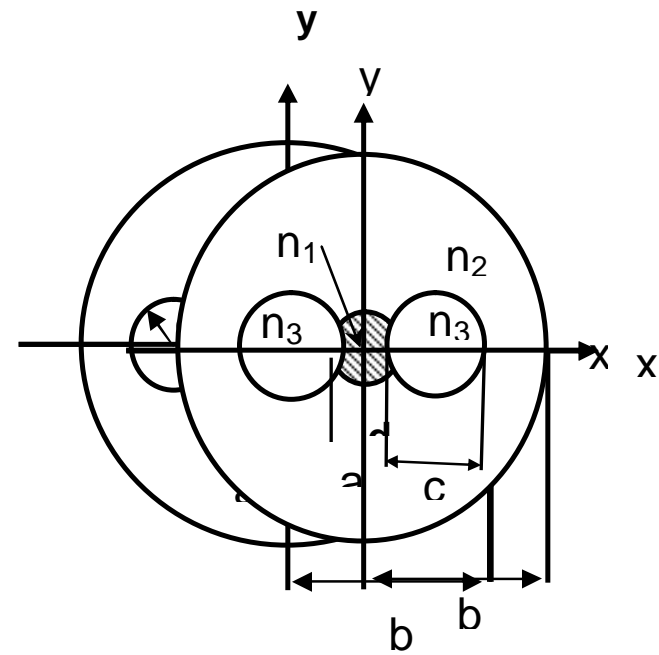

Fig. 2 Panda fiber

Figure 3 shows the stress distribution $\sigma_{\mathrm{x}}$ and $\sigma_{\mathrm{y}}$ on the $\mathrm{x}$-axis of the Panda type fiber when force is applied along the $y$-axis. It can be observed from the curve that stresses

Table I Parameters of the Panda and the Side Tunnel fibers 


\begin{tabular}{|c|c|c|}
\hline & $\begin{array}{c}\text { Side Tunnel } \\
\text { fiber }\end{array}$ & Panda fiber \\
\hline $2 \mathrm{a}(\mu \mathrm{m})$ & $5 \sim 10$ & 10 \\
\hline $2 \mathrm{~b}(\mu \mathrm{m})$ & 125 & 120 \\
\hline$\alpha_{1}\left({ }^{\circ} \mathrm{C}^{-1}\right)$ & $7.6 \times 10^{-6}$ & $2.125 \times 10^{-6}$ \\
$\alpha_{2}\left({ }^{\circ} \mathrm{C}^{-1}\right)$ & $5.4 \times 10^{-7}$ & $5.4 \times 10^{-7}$ \\
$\alpha_{3}\left({ }^{\circ} \mathrm{C}^{-1}\right)$ & $3.66 \times 10^{-3}$ & $1.45 \times 10^{-6}$ \\
\hline $\mathrm{d} / \mathrm{c}$ & 0.15 & - \\
$\mathrm{a} / \mathrm{d}$ & $2.0 \sim 4.0$ & - \\
\hline $\mathrm{E}\left(\mathrm{kg} / \mathrm{mm}^{2}\right)$ & 7830 & 7830 \\
\hline$v$ & 0.186 & 0.186 \\
\hline $2 \mathrm{c}(\mu \mathrm{m})$ & - & 30 \\
\hline $\mathrm{d}_{1}(\mu \mathrm{m})$ & - & 20 \\
\hline $\mathrm{d}_{2}(\mu \mathrm{m})$ & - & 35 \\
\hline
\end{tabular}

are nearly constant in the core region of the fiber. For the clad region between the core region and the SAZ region, the first clad region, stresses decrease gradually. Reaching the SAZ, it is observed that the stresses nearly equal zero. The behavior of the stresses in the core and the first clad region can be explained by the resultant stresses because external and internal stresses existing at these regions are not equal and the net stress varies uniformly as we proceed along the fiber cross section. After this region, the stresses are nearly equal resulting in a nearly zero stress.

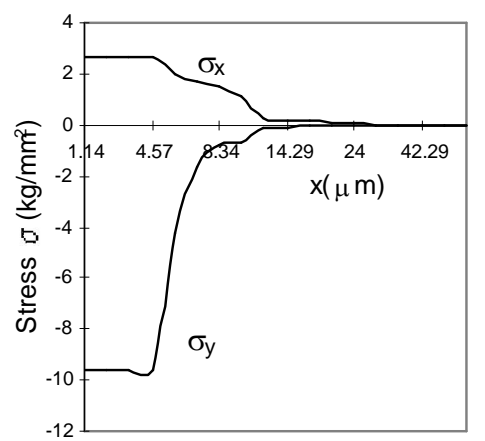

Fig. 3 Stress distribution on the $x-$ axis when force is applied along $y-$ axis in the Panda fiber.

Figure 4 shows the stress distribution on the y-axis when force is applied along the $\mathrm{x}$-axis in the Panda fiber. One can observe the same behavior as in Fig. 3. As we reach the clad region, one observes that stresses decrease gradually. The decrement of the stresses along the y-axis can be explained by the decrement of the external stresses because the area is affected by the external normal force along the axis that increases when proceeding along the axis. The applied load, $\mathrm{W}_{\mathrm{o}}$, in both Figs. 3 and 4 is $0.5 \mathrm{~kg} / \mathrm{cm}$.
Based on Ref. [8] and stresses shown in Fig. 3, the stress-induced modal birefringence in the Panda type fiber when force is applied along the $\mathrm{x}$-axis direction is shown in Fig. 5. It is observed from the figure that the modal birefringence is directly proportional to the radial distance from the fiber center. This is due to the effect of the stresses at this region. The value of the birefringence also depends on the magnitude of the external applied forces. Similarly, the stress-induced modal birefringence when force is applied along the yaxis is shown in Fig. 6. The figure shows nearly the same behavior as in Fig. 5. It is clear that the birefringence at this applied load equals zero at the core region.

Changing the applied load, $\mathrm{W}_{\mathrm{o}}$, the stress difference, $\sigma_{\mathrm{x}}-\sigma_{\mathrm{y}}$, is calculated when the force is applied in $\mathrm{x}$ - and $y$ - directions, respectively. From this difference, the stress-induced modal birefringence is obtained.

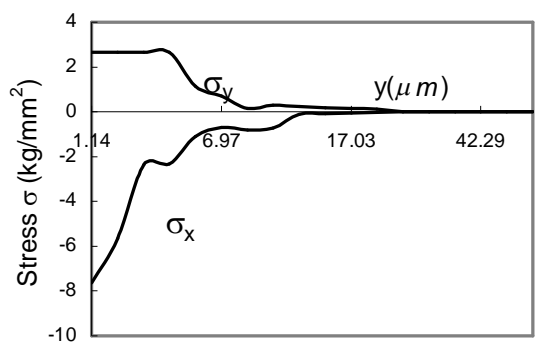

Fig. 4 Stress distribution on the $y$ axis when force is applied along $\mathrm{x}$ axis in the Panda fiber.

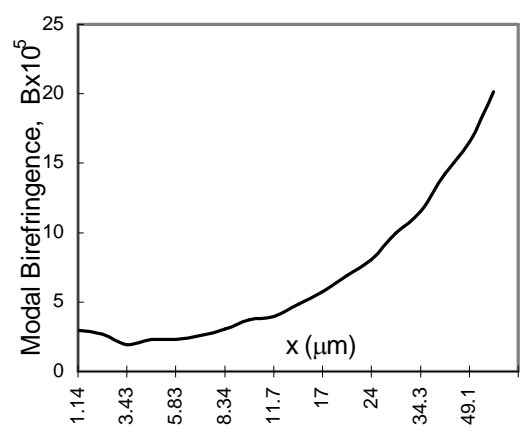

Fig. 5 Modal birefringence in the Panda fiber when force is applied along $\mathrm{X}$-axis. 


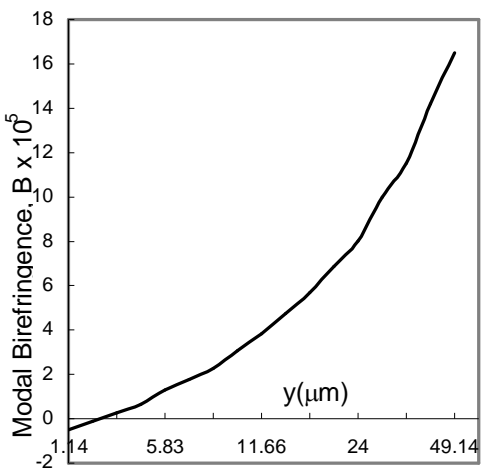

Fig. 6 Modal birefringence in the Panda fiber when force is applied along y-axis direction.

The stress difference in the Panda fiber when force is applied along $\mathrm{x}$-axis is shown in Fig. 7, from which it is noted that the stress difference decreases with the applied loads on the fiber. This means that the stress along the $y$-axis is greater than the stress along the $\mathrm{x}$ axis. Figure 8 shows the modal birefringence in the Panda fiber when force is applied along the $\mathrm{x}$-axis. It is clear that the modal birefringence decreases with the applied loads on the fiber.

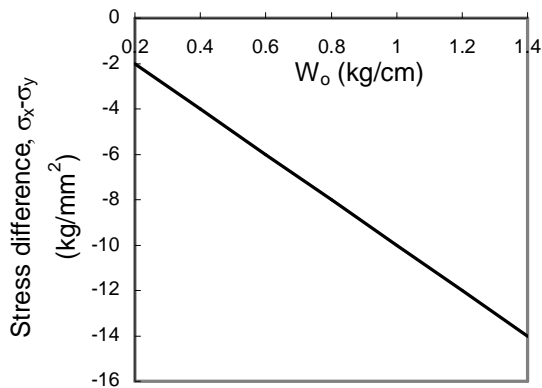

Fig. 7 Stress difference in the Panda fiber when force is applied along the $\mathrm{X}$-axis.

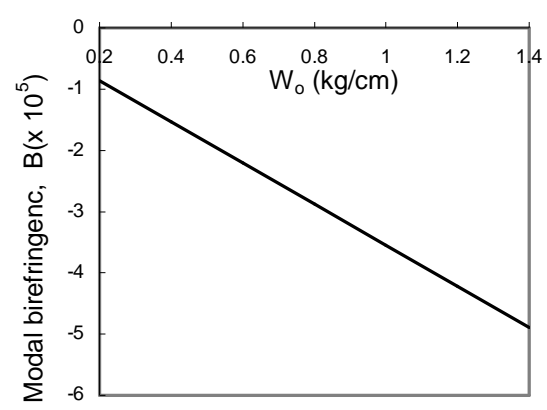

Fig. 8 Modal birefringence in the Panda fiber when force is applied along $\mathrm{X}$-axis.

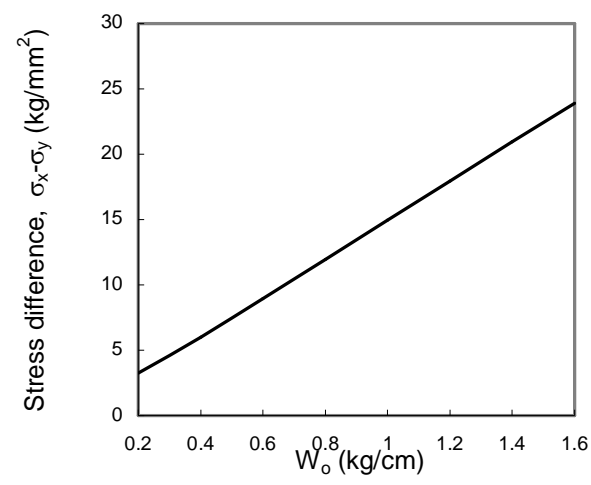

Fig. 9 Stress distribution in the Panda fiber when force is applied along $\mathrm{y}$-axis.

Figure 9 shows the stress distribution in the Panda fiber when force is applied along the y-axis. A direct proportionality between the applied loads, $\mathrm{W}_{\mathrm{o}}$, and the stress difference is noticed. Figure 10 shows the modal birefringence in the Panda fiber when force is applied along the y-axis, where an increase is noticed in the modal birefringence with the applied loads. From these results it is expected that we can control the magnitude of the birefringence in the fiber by controlling the external loads acting on the fiber.

Figures 11 and 12 show the stress distribution for the Side Tunnel fiber on the $\mathrm{x}$-axis and the $\mathrm{y}$-axis directions. The two figures show nearly the same behavior and the same order of magnitude as the Panda fiber. This is expected since the two fibers are different only in the position of the SAZs and the parameters

This is repeated for the modal birefringence, Fig. 13 and 14. As the force is applied along the y-direction in the fiber, one observes that the modal birefringence vanishes nearly at the limit of the fiber. It is also expected that the modal birefringence will vanish as the magnitude of the external applied loads, $W_{o}$, is varied. Similar to the Panda fiber, the behavior of the Side Tunnel fiber is shown in Figs. 15 to 18.

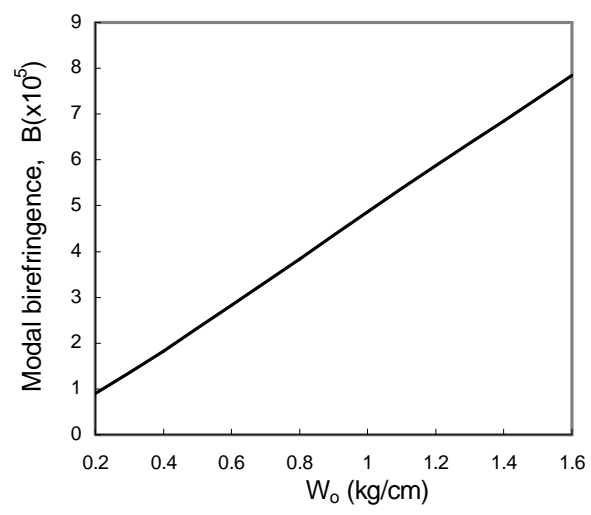

Fig. 10 Modal birefringence in the Panda fiber when force is applied along $\mathrm{y}$-axis. 


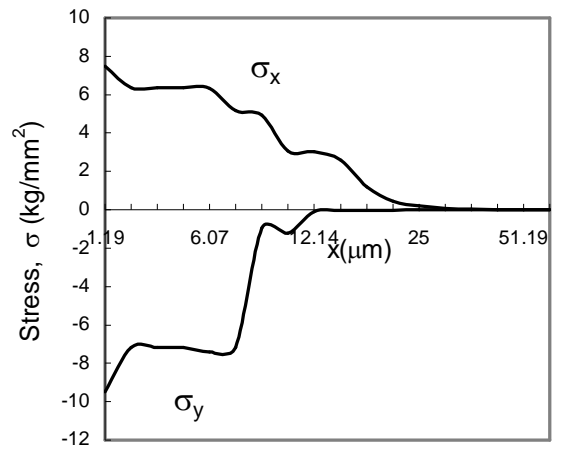

Fig.11 Stress distribution for the Side Tunnel fiber on the $\mathrm{x}$-axis when force is applied along $\mathrm{y}$-axis.

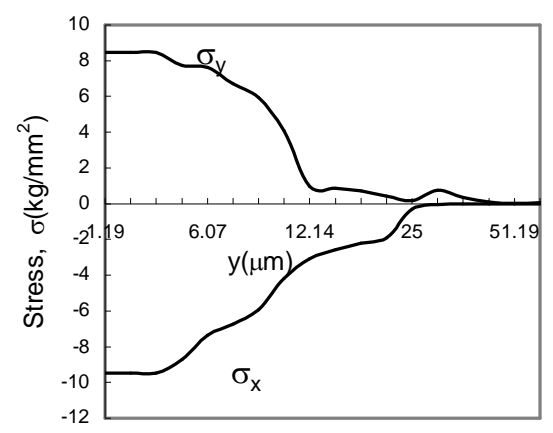

Fig. 12 Stress distribution for the Side Tunnel fiber on the $y$-axis when force is applied along $\mathrm{x}$-axis.

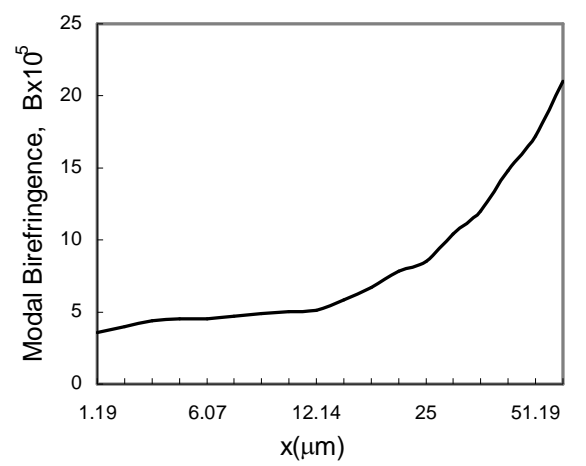

Fig. 13 Modal birefringence in the Side Tunnel fiber when force is applied along the $\mathrm{x}$-axis.

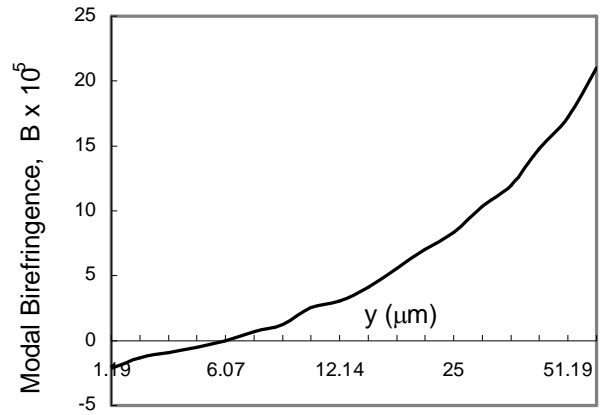

Fig. 14 Modal birefringence in the Side Tunnel fiber when force is applied along the $\mathrm{y}$-axis.

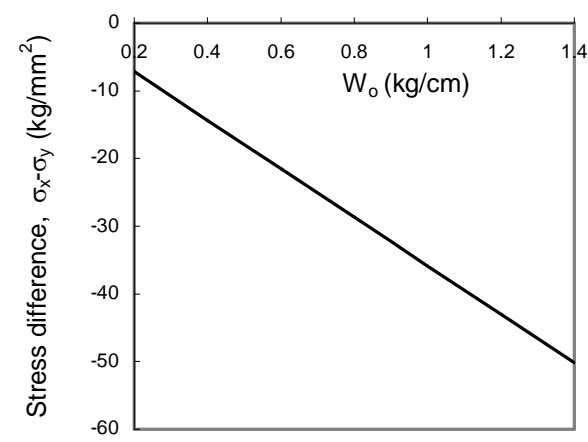

Fig. 15 Stress difference in the Side Tunne fiber when force is applied along $\mathrm{x}$-axis.

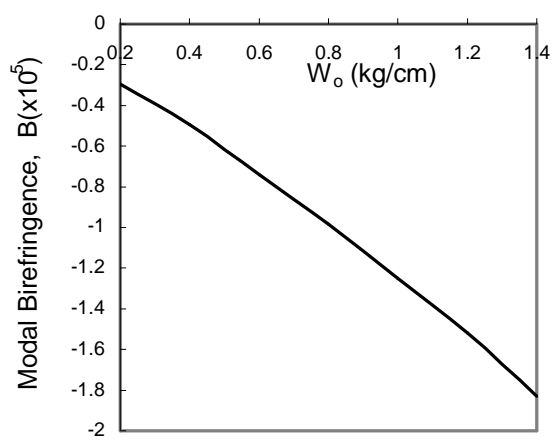

Fig. 16 Modal birefringence in the Sid $\epsilon$ Tunnel fiber when force is applied along $\mathrm{x}$. axis. 


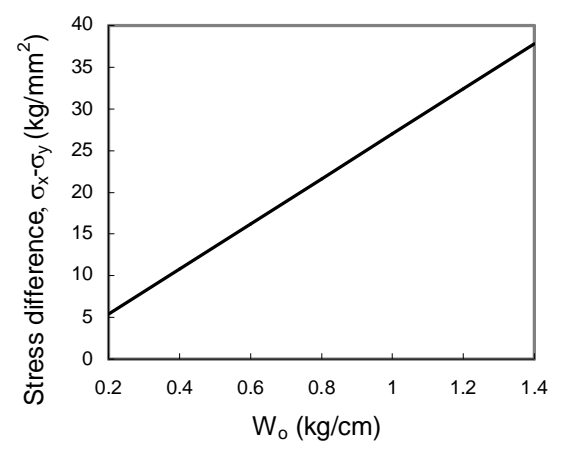

Fig. 17 Stress difference in the Side Tunnel fiber when force is applied along $\mathrm{y}$-axis.

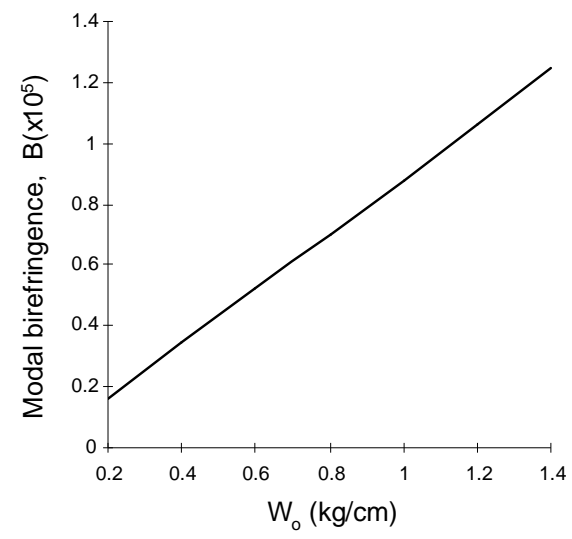

Fig 18 Modal birefringence in the Side Tunnel fiber when force is applied along the y-axis.
[2] S. C. Chao, "Extended Gaussian Approximation for Single-Mode Graded Index Fibers". IEEE J. Lightwave Technol., Vol. LT-12 (3), pp. 392-395, 1994.

[3] D. Marcuse and C. Lin, "Low Dispersion Single-Mode Fiber Transmission", IEEE J. Quantum Electron, Vol. QE-17, No. 6, pp. 869-877,1981.

[4] C. X. Sho and U R.Q. Hui, "Polarization Coupling in Single-Mode Single-Polarization Fibers", Opt. Lett., Vol. 13, No. 12, pp. 1120-1122, 1988.

[5] P. K. Bachmann, Dieter Leers, Hermann Wehr and Erich R. Wehrhahn, "Dispersion-Flattened Single Mode Fibers prepared with PCVD: Performance, Limitations, Design Optimization", IEEE J.Lightwave Technol., Vol. LT-4 (7), pp. 858-863, 1983.

[6] K. T. Bathe, Finite Element Procedures, Prentice Hall Inc., 1996.

[7] B. M. A. Rahaman, A. Fernandez, and B. Davies, "Review of Finite Element Methods for Microwave and Optical Waveguides", IEEE J. Lightwave Technol., Vol. LT-13, pp. 1442-1446, 1991.

[8] K. Hayata and M. Koshiba, "Stress-induced birefringence of Side-Tunnel Type PolarizationMaintaining Fiber", IEEE J. Lightwave Technol., Vol. LT-4, 1986.

\section{CONCLUSION}

The finite element method has been applied for both the Side Tunnel and Panda fibers to obtain variation in stresses and modal birefringence.

It is found that the modal birefringence is directly proportional to radial distance from the fiber center. It is also found that as the force is applied, the modal birefringence vanishes nearly at the core limits of the fiber.

\section{REFERENCES}

[1] K. Okamoto, T. Hosaka and T. Edahiro, "Stress Analysis of Optical Fibers by a Finite Element Method", IEEE J. Quantum Electron, Vol. QE-17, No. 10, pp. 2123-2129, 1981. 


\section{BIOGRAPHY}

Prof. Dr. Moustafa Hussein Aly is currently Professor of Engineering Physics, Faculty of Engineering, University of Alexandria, Alexandria, Egypt. He was born in Alexandria in 1953. He received his B.Sc. in 1976 in Communications and Electrophysics, his M.Sc. in 1983 and his Ph.D. in 1987 in Engineering Physics, all from Faculty of Engineering, University of Alexandria, Egypt. He is a member of the Optical Society of America (OSA) and of the Egyptian Society of Solid State (ESSS). His area of interest is Laser and Fiber Optics where he has about 40 publications. E-mail: mosaly@hotmail.com
Mr. Ashraf M. S. Farahat is currently a lecturer, Department of Physics, Faculty of Science, University of Alexandria, Alexandria, Egypt. He was born in Alexandria in 1972. He received his B.Sc. in 1992 and M.Sc. in 1999, both in Physics, Faculty of Science, University of Alexandria, Egypt.

Dr. Maher Farhoud is currently Associate Professor, Department of Physics, Faculty of Science, University of Alexandria, Alexandria, Egypt. He was born in Alexandria in 30/11/1958. He received his B.Sc. in 1980 and M.Sc. in 1986, both in Physics, Faculty of Science, University of Alexandria, Egypt. He received his Ph.D. in Physics, Adam Mickiwicz University, Poland. His area of interest is Laser and Nonlinear Optics. E-mail: mfarhoud@yahoo.com. 